\title{
Post stroke hemi-dystonia in children: a neglected area of research
}

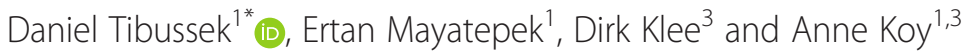

\begin{abstract}
Background: Childhood arterial ischemic stroke (CAIS) is increasingly recognized as an important cause of significant long-term morbidity in the pediatric population. Post stroke movement disorders, above all hemi-dystonias, are much more common in children after stroke compared to adults. However, research in this field is largely lacking. By highlighting some important knowledge gaps, we aim to encourage future collaborative research projects in this particular field.

Findings: Post stroke-dystonia seems to be much more common among children than adults. However, no reliable epidemiological data of post-stroke movement disorders in childhood are available, and differentiation between spasticity and dystonia can be challenging. Pharmacotherapy for dystonia is limited by lack of effect, especially in the long-term treatment. The pathophysiology of dystonia is complex and incompletely understood. Recent findings from functional imaging studies suggest that dystonia does not result from a single lesion but rather network dysfunctions and abnormalities in functional connectivity. However, very few patients with post stroke dystonia have been studied, and it is not clear to what extent pathophysiology of primary and post stroke ischemia shares common characteristics on network level. In general, progress in understanding the nature of childhood dystonia lags far behind adult onset CNS diseases.

Conclusions: Dystonia after CAIS is a common yet insufficiently understood and poorly studied clinical challenge. Studies to improve our understanding of the underlying pathophysiology and consequently the development of instruments for early prediction as well as targeted treatment of dystonia should become a high priority in collaborative childhood stroke research.
\end{abstract}

Keywords: Childhood stroke, Basal ganglia, Dystonia, Movement disorders, Functional imaging, Transcranial magnetic stimulation

\section{Background}

Childhood arterial ischemic stroke (CAIS) is an important cause of morbidity and mortality in the pediatric population. With an estimated incidence of 1.6 per 100,000 children per year (excluding neonates; [1]), more than 200 new cases can be expected to occur in Germany each year. Early brain injury due to stroke commonly results in significant long-term impairment [2-5]. Hemi-dystonia is a common, yet insufficiently studied motor problem after CAIS leading to significant life-long disability [6].

\footnotetext{
*Correspondence: daniel.tibussek@gmx.net

'Department of General Pediatrics, Neonatalogy and Pediatric Cardiology, University Children's Hospital, Heinrich-Heine University, Moorenstrasse 5, 40225 Düsseldorf, Germany

Full list of author information is available at the end of the article
}

The purpose of this review is to summarize current knowledge about post stroke dystonia. By highlighting some important knowledge gaps, we further aim to encourage future collaborative research projects.

\section{Definition}

According to a recent consensus update, "dystonia is a movement disorder characterized by sustained or intermittent muscle contractions causing abnormal, often repetitive, movements, postures, or both. Dystonic movements are typically patterned, twisting, and may be tremulous. Dystonia is often initiated or worsened by voluntary action and associated with overflow muscle activation [involuntary activation of muscles that are not required to perform a given movement]" [7]. Dystonia can be focal, segmental, multifocal, or generalized. Hemi-dystonia is defined as dystonia

\section{Springer}


involving the ipsilateral arm and leg with or without affecting the face, neck, or trunk. The former classification into "primary" and "secondary" dystonia is currently discouraged. The new etiological classification now includes pattern of inheritance and nervous system pathology [7].

\section{Epidemiology}

At present, no reliable epidemiological data of post stroke movement disorders in childhood are available. In adults, post stroke movement disorders in general are considered rare, affecting only $1 \%$ of stroke patients [8].

Post stroke-dystonia seems to be much more common among children than adults [6]. A Canadian study found that $21 \%$ of the children with basal ganglia strokes will eventually develop dystonia [9]. This stroke pattern is particularly common in children with focal cerebral arteriopathy (FCA), an important, presumably inflammatory cause of CAIS (Fig. 1). FCA is a unilateral arteriopathy of the large vessels of the anterior circulation, typically affecting the distal ICA and proximal segments of the middle cerebral artery (MCA) and anterior cerebral artery (ACA) [10]. Cerebral infarcts due to FCA are nearly always located in perforator territories within the basal ganglia zone.

\section{Clinical characteristics and evaluation}

Post stroke dystonia is usually unilateral, contralateral to the brain lesion; however, it can also be bilateral or generalized. Rarely, ipsilateral dystonia has been described [11]. Post stroke dystonia mostly involves the distal upper limb and often severely impairs manual functions $[6,12]$. It can occur immediately at stroke onset (rare) or, typical in children, can be delayed by months or even years [13]. This is in contrast to post stroke spasticity, which typically evolves within the first few days or weeks post stroke. The reason for the delay of clinical manifestation is incompletely understood. Age-related maladaptive neuroplasticity as well as different CNS structures and networks involved is likely to play a role. Future functional imaging studies will help to better understand the underlying network damages.

Assessing dystonia is challenging because of its dynamic nature and overlap with other types of movement disorders [14]. One study on motor impairment after childhood onset stroke found that 15 of 24 $(62.5 \%)$ children with hemiparesis after stroke had additional dystonia [15]. Diagnosing dystonia in these cases is challenging; however, diagnostic instruments to assist with the differentiation between spasticity and dystonia are available [16]. These instruments enable to distinguish between involuntary movements or greater tone with purposeful movements (dystonia) or increased resistance during fast stretch compared to low stretch (spasticity).

Assessments capturing the impact of dystonia on motor function, activities of daily living, and the child's ability to participate have also been invented [7]. Additional kinematic analyses (joint position, velocity, and acceleration of motion) can add important quantitative information about the functional severity more objectively $[7,17]$.

\section{Treatment dilemmas}

Hemi-dystonia almost always has severe impact on motor function; however, response to treatment is typically poor [17]. Pharmacotherapy for dystonia is limited by lack of effect, especially in the long-term treatment, or because side effects are not tolerated ([17] Table 1). Botulinum toxin A injections in affected muscles can reduce painful spasms but do usually not directly improve function [17, 18]. Meanwhile, physiotherapy and occupational therapy are accepted as essential components to a multidisciplinary therapy; research in this field is particularly limited. Constraint induced movement therapy (CIMT), an established rehabilitation strategy after adult stroke and for



Fig. 1 Right basal ganglia stroke (a) due to a focal cerebral arteriopathy involving the M1 segment of the right middle cerebral artery (MCA). Note the beading appearance of the affected MCA segment $(\mathbf{b})$ 
Table 1 Current and emerging treatment options for dystonia [16, 17]

\begin{tabular}{ll}
\hline Oral & - Anticholinergics (e.g., trihexyphenidyl)- Tetrabenazine- L-dopa/carbidopa- Baclofen- Benzodiazepines (e.g., diazepam, \\
& clonazepam)- Muscle relaxants (e.g., clonidin)- others (e.g., amitriptyline, gabapentin) \\
Intramuscular & - Botulinum toxin \\
Intrathecal & - Baclofen \\
Behavioral & - Biofeedback- Constraint induced therapy \\
(Noninvasive) & - Transcranial magnetic stimulation \\
neuromodulation & \\
(Invasive) & - Deep brain stimulation \\
neuromodulation & \\
\hline
\end{tabular}

unilateral cerebral palsy $[19,20]$, has not yet been sufficiently evaluated in children with dystonia. A single pilot study evaluated the effect of CIMT in six children after stroke, including three with dystonia, with positive effects on functional performance [21].

Therefore, more studies and further treatment options are urgently needed.

Recently, biofeedback and transcranial magnetic stimulation (TMS) have gained some attention as noninvasive dystonia therapies. TMS is a painless method to stimulate the human brain and modulate its excitability [22]. Encouraging data from patients with writer's cramp and the noninvasiveness of this technique make TMS an attractive candidate for future clinical studies in childhood [22].

Biofeedback studies focusing on inappropriate muscle activation and force adoption suggest that visual biofeedback of muscle activity can help to reduce excess muscle activation [23]. Children with dystonia may therefore to some extent be able to control co-contraction and reduce overflow by these strategies [17].

Deep brain stimulation (DBS) of the globus pallidus internus can be an effective and safe treatment option for inherited isolated generalized, segmental, and cervical dystonia with improvement in the Burke-Fahn-Marsden Dystonia Rating Scale (BFMDRS) of up 60-90 \% [24], whereas the outcome for patients with acquired dystonia such as dyskinetic cerebral palsy is less distinctive and more heterogeneous, with a mean improvement of about $24 \%$ [25]. The experience with uni- or bilateral DBS for pediatric patients with structural lesions due to stroke or traumatic brain injury is very limited to a few small case series [26, 27]. It is of note, that despite little or missing improvement in motor scores, patients report about other beneficial effects such as reduction of pain or decrease of muscle tone [27]. Therefore, despite little evidence to support this therapeutic option, DBS should be considered for young patients with disabling dystonia in the absence of other effective treatment options.

Future studies on greater (pediatric) cohorts are urgently needed to evaluate the effects of DBS in acquired forms of dystonia such as post stroke dystonia comprehensively and to identify prognostic markers for the therapeutic effect.

\section{Functional anatomy of basal ganglia}

The pathophysiology of dystonia is complex and incompletely understood.

Commonly assumed models of basal ganglia dysfunction in dystonia are certainly oversimplified. However, they help to understand some basic ideas behind pathophysiology and also treatment.

In the majority of cases of post stroke dystonia, lesions in the basal ganglia, especially putamen, are found [11]. However, multiple other stroke locations have been associated, including thalamus, caudate, internal capsule, brainstem, cerebellum, and spinal cord [11]. Recent findings from functional imaging studies suggest that dystonia does not result from a single lesion but rather network dysfunctions and abnormalities in functional connectivity [28, 29]. Meanwhile, the basal ganglia and related thalamo-cortical networks are major determinants in the pathophysiology, sensorimotor cortex, and alterations of cerebello-thalamo-cortical pathways likely play an additional important role [28, 29].

Different resulting pathophysiological alterations have been suggested:

1. Defects of inhibitory circuits at the spinal, brainstem, cerebellar, or cortical level with decreased inhibition of unwanted muscle patterns

2. Abnormal sensory function and sensorimotor integration

3. Maladaptive plasticity of the sensorimotor cortex

However, it is increasingly recognized that different forms of dystonia have a different neuroanatomical origin, and results of recent electrophysiological and functional imaging studies of dystonia are not always consistent [28, 29]. Very few patients with post stroke dystonia have been studied. It is yet not clear to what extent pathophysiology of idiopathic or genetic and post stroke dystonia share common characteristics at network level [30-32]. 
After CAIS, it can be assumed that network changes differ significantly depending on the age at the time of the stroke event. Maladaptive plasticity may play a major role as animal studies of motor function outcome after stroke suggest that effects of plasticity can impair motor recovery by leading to network dysfunctions [33].

\section{Cerebral networks and modern imaging: missed opportunities?}

With the progress of modern brain imaging techniques more detailed analyses of type, localisation and extent of ischemic lesions as well as cerebral reorganization and functional consequences after cerebral ischemia have been made possible. Along with the improved understanding of network dysfunctions, early prediction of dystonia and more specific treatment approaches may be available in the future. Nevertheless, comparing the enormous number of functional imaging studies in adults with (mostly inherited isolated) dystonia with the very limited research in children highlights obvious difficulties and challenges of studying children (Table 2). Progress in understanding the nature of childhood dystonia lacks far behind adult onset CNS diseases.

An increasing variety of (functional) imaging methods is available. Recently, resting state (rs) fMRI, the examination of spontaneous brain function by using blood oxygen level-dependent contrast in the absence of a task, has facilitated noninvasive mapping of neural network dysfunction even in children [34]. Using spontaneous activity, resting state maps can be generated reflecting functional brain organization. Limited $r s$ fMRI data is available on dystonia [35, 36]. Abnormal functional connectivity was found in patients with writer's cramp as well as cervical dystonia. Preliminary data suggest that this technique can be successfully used in disabled, asleep, or even sedated children with scanning times of about 5 min [34].

Magnetoencephalography (MEG) might be another noninvasive method for network studies in children. MEG is a technique that records magnetic fields generated by the brain. Because it is also possible to do depth recording, three-dimensional information can be gathered. MEG studies in patients with focal dystonias show considerable overlap with findings from neuroimaging studies indicating reduced inhibition and disturbed sensory-motor integration [37]. Future research will be needed to proof its reliable use in children.

\section{Conclusion and future directions}

Dystonia after CAIS is a common yet insufficiently understood and poorly studied clinical challenge. Population-based studies are needed to better define prevalence, clinical presentation, time course, and
Table 2 Some pediatric challenges in functional imaging studies [28]

Small patient numbers
Limited normative data
Child unfriendly environment (MRI)
Acoustic noise
Motion artifacts
Effects of maturation
Effects of sedation
Limited cooperation
Monitoring task performance
Limited acquisition time

treatment response of pediatric post stroke dystonia. Studies using modern, innovative imaging techniques will help to improve our understanding of the underlying pathophysiology and consequently the development of instruments for early prediction as well as targeted treatment of dystonia. Finally, treatment studies should evaluate whether noninvasive treatments such as TMS or biofeedback have the potential to improve motor function and quality of life in children with post stroke dystonia.

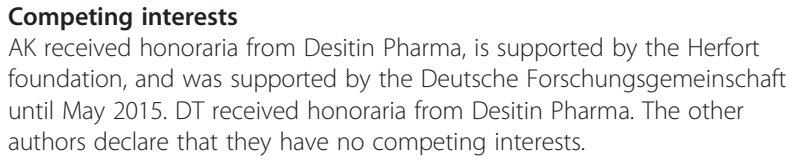

\section{Authors' contributions}

DT initiated the review, designed the structure of the review, did the literature search, and prepared the first draft and subsequent versions of the review. AK did the literature search, modified the first and subsequent drafts, and contributed to the preparation of tables. EM and DK contributed to writing, and critically revised the draft. All authors read and approved the final manuscript.

\section{Author details}

${ }^{1}$ Department of General Pediatrics, Neonatalogy and Pediatric Cardiology, University Children's Hospital, Heinrich-Heine University, Moorenstrasse 5, 40225 Düsseldorf, Germany. ${ }^{2}$ Department of Diagnostic and Interventional Radiology, Faculty of Medicine, University Dusseldorf, Heinrich-Heine University, Moorenstrasse 5, 40225 Düsseldorf, Germany. ${ }^{3}$ Department of Neurology, University of Cologne, Kerpener Strasse 62, 50924 Cologne, Germany.

Received: 10 June 2015 Accepted: 8 December 2015

Published online: 11 December 2015

\section{References}

1. Mallick AA, Ganesan V, Kirkham FJ et al (2014) Childhood arterial ischaemic stroke incidence, presenting features, and risk factors: a prospective population-based study. Lancet Neurol 13(1):35-43

2. Ganesan V, Hogan A, Shack N, Gordon A, Isaacs E, Kirkham FJ (2000) Outcome after ischaemic stroke in childhood. Dev Med Child Neurol 42:455-461

3. deVeber G, MacGregor D, Curtis R, Mayank S (2000) Neurologic outcome in survivors of childhood arterial ischemic stroke and sinovenous thrombosis. J Child Neurol 15:316-324

4. Steinlin M, Pfister I, Pavlovic J, Everts R, Boltshauser E, Capone Mori A et al (2005) The first three years of the Swiss Neuropaediatric Stroke Registry (SNPSR): a 
population-based study of incidence, symptoms and risk factors. Neuropediatrics 36:90-97

5. Christerson S, Stromberg B (2010) Stroke in Swedish children II: long-term outcome. Acta Paediatr 99:1650-1656

6. Béjot Y, Giroud M, Moreau T, Benatru I (2012) Clinical spectrum of movement disorders after stroke in childhood and adulthood. Eur Neurol 68:59-64

7. Albanese A, Bhatia K, Bressman SB et al (2013) Phenomenology and classification of dystonia: a consensus update. Mov Disord 28:863-873

8. Ghika-Schmid F, Ghika J, Regli F, Bogousslavsky J (1997) Hyperkinetic movement disorders during and after acute stroke: the Lausanne Stroke Registry. J Neurol Sci 10(146):109-116

9. Soman T, Askalan R, Martin M, Allen A, Zak M, MacGregor D, Logan W (2006) Predictors of dystonia in childhood basal ganglia stroke. Neuropediatrics 37 - TP121. DOI: 10.1055/s-2006-945715

10. Bernard TJ, Manco-Johnson MJ, Lo W et al (2012) Towards a consensus-based classification of childhood arterial ischemic stroke. Stroke 43:371-377

11. Mehanna R, Jankovic J (2013) Movement disorders in cerebrovascular disease. Lancet Neurol 12:597-608

12. de Campos AC, Kukke SN, Hallett M, Alter KE, Damiano DL (2014) Characteristics of bilateral hand function in individuals with unilateral dystonia due to perinatal stroke: sensory and motor aspects. J Child Neurol 29:623-632

13. Scott B, Jankovic J (1996) Delayed-onset progressive movement disorders after static brain lesions. Neurology 46:68-74

14. Pavone L, Burton J, Gaebler-Spira D (2013) Dystonia in childhood: clinical and objective measures and functional implications. J Child Neurol 28(3):340-350

15. Boardman JP, Ganesan V, Rutherford MA, Saunders DE, Mercuri E, Cowan F (2005) Magnetic resonance image correlates of hemiparesis after neonatal and childhood middle cerebral artery stroke. Pediatrics 115(2):321-326

16. Jethwa A, Mink J, Macarthur C, Knights S, Fehlings T, Fehlings D (2010) Development of the Hypertonia Assessment Tool (HAT): a discriminative tool for hypertonia in children. Dev Med Child Neurol 52:e83-e87

17. Bertucco M, Sanger TD (2015) Current and emerging strategies for treatment of childhood dystonia. J Hand Ther 28(2):185-194

18. Roubertie A, Mariani LL, Fernandez-Alvarez E, Doummar D, Roze E (2012) Treatment for dystonia in childhood. Eur J Neurol 19:1292-1299

19. Kwakkel G, Veerbeek JM, van Wegen EE, Wolf SL (2015) Constraint-induced movement therapy after stroke. Lancet Neurol 14(2):224-234

20. Sakzewski L, Ziviani J, Boyd RN (2014) Efficacy of upper limb therapies for unilateral cerebral palsy: a meta-analysis. Pediatrics 133(1):e175-e204

21. Gordon A, Connelly A, Neville B, Vargha-Khadem F, Jessop N, Murphy T, Ganesan V (2007) Modified constraint-induced movement therapy after childhood stroke. Dev Med Child Neurol 49:23-27

22. Quartarone A (2013) Transcranial magnetic stimulation in dystonia. Handb Clin Neurol 116:543-553

23. Bloom R, Przekop A, Sanger TD (2010) Prolonged electromyogram biofeedback improves upper extremity function in children with cerebral palsy. J Child Neurol 25:1480e1484

24. Kupsch A, Benecke R, Müller J, Deep-Brain Stimulation for Dystonia Study Group et al (2006) Pallidal deep-brain stimulation in primary generalized or segmental dystonia. N Engl J Med 355:1978-1990

25. Koy A, Hellmich M, Pauls KA et al (2013) Effects of deep brain stimulation in dyskinetic cerebral palsy: a meta-analysis. Mov Disord 28(5):647-654

26. Air EL, Ostrem JL, Sanger TD, Starr PA (2011) Deep brain stimulation in children: experience and technical pearls. J Neurosurg Pediatr 8:566-574

27. Slow EJ (2015) Deep brain stimulation for treatment of dystonia secondary to stroke or trauma. J Neurol Neurosurg Psychiatry 86:1046-1048

28. Lehéricy S, Tijssen MA, Vidailhet M, Kaji R, Meunier S (2013) The anatomical basis of dystonia: current view using neuroimaging. Mov Disord 15(28):944-957

29. Stoessl AJ, Lehericy S, Strafella AP (2014) Imaging insights into basal ganglia function, Parkinson's disease, and dystonia. Lancet 9(384):532-534

30. Ceballos-Baumann AO, Passingham RE, Marsden CD, Brooks DJ (1995) Motor reorganization in acquired hemidystonia. Ann Neurol 37:746-757

31. Lehéricy S, Gerardin E, Poline JB, Meunier S, Van de Moortele PF, Le Bihan D, Vidailhet M (2004) Motor execution and imagination networks in post-stroke dystonia. Neuroreport 26(15):1887-1890

32. Kojovic M, Pareés I, Kassavetis P, Palomar FJ, Mir P, Teo JT, Cordivari C, Rothwell JC, Bhatia KP, Edwards MJ (2013) Secondary and primary dystonia: pathophysiological differences. Brain 136:2038-2049
33. Jones TA, Allred RP, Jefferson SC, Kerr AL, Woodie DA, Cheng SY, Adkins DL (2013) Motor system plasticity in stroke models: intrinsically use-dependent, unreliably useful. Stroke 44:S104-S106

34. Hertz-Pannier L, Noulhiane M, Rodrigo S, Chiron C (2014) Pretherapeutic functional magnetic resonance imaging in children. Neuroimaging Clin N Am 24(4):639-653

35. Delnooz CC, Pasman JW, Beckmann CF, van de Warrenburg BP (2013) Taskfree functional MRI in cervical dystonia reveals multi-network changes that partially normalize with botulinum toxin. PLoS ONE 1(8):e62877

36. Mohammadi B, Kollewe K, Samii A, Beckmann CF, Dengler R, Munte TF (2011) Changes in resting-state brain networks in writer's cramp. Hum Brain Mapp 33:840-848

37. Zoons E, Booij J, Nederveen AJ, Dijk JM, Tijssen MA (2011) Structural, functional and molecular imaging of the brain in primary focal dystonia-a review. Neuroimage 56:1011-1020

\section{Submit your manuscript to a SpringerOpen ${ }^{\circ}$ journal and benefit from:}

- Convenient online submission

- Rigorous peer review

- Immediate publication on acceptance

- Open access: articles freely available online

- High visibility within the field

- Retaining the copyright to your article

Submit your next manuscript at $\gg$ springeropen.com 